\title{
A Novel Method for Preparation of Zn-Doped CuInS 2 Solar Cells and Their Photovoltaic Performance
}

\author{
Cheng-Hsiung Peng ${ }^{1}$ and Chyi-Ching Hwang ${ }^{2}$ \\ ${ }^{1}$ Department of Chemical and Materials Engineering, Minghsin University of Science and Technology, \\ Hsinfeng, Hsinchu 304, Taiwan \\ ${ }^{2}$ Weapon System Center, Chung Cheng Institute of Technology, NDU, Daxi, Taoyuan 335, Taiwan \\ Correspondence should be addressed to Cheng-Hsiung Peng; chpeng@must.edu.tw
}

Received 3 August 2013; Accepted 30 September 2013

Academic Editors: K. Máthis and Z. Peng

Copyright (C) 2013 C.-H. Peng and C.-C. Hwang. This is an open access article distributed under the Creative Commons Attribution License, which permits unrestricted use, distribution, and reproduction in any medium, provided the original work is properly cited.

In this study, a novel method was proposed to synthesize high quality Zn-doped $\mathrm{CuInS}_{2}$ nanocrystals under high frequency magnetic field at ambient conditions. The magnetic Zn-doping gave superparamagnetic heating of the resulting nanocrystals via magnetic induction, causing an accelerating growth rate of the doped $\mathrm{CuInS}_{2}$ under ambient conditions faster than conventional autoclave synthesis. Shape evolution of the $\mathrm{Zn}$-doped $\mathrm{CuInS}_{2}$ nanocrystals from initially spherical to pyramidal, to cubic, and finally to a bar geometry was detected as a function of time of exposure to magnetic induction. These colloidal solvents with different shaped nanocrystals were further used as "nanoink" to fabricate a simple thin film solar device; the best efficiency we obtained of these crystals was $1.01 \%$ with a $1.012 \mu \mathrm{m}$ thickness absorber layer (bar geometry). The efficiency could be promoted to $1.44 \%$ after the absorber was thickened to $2.132 \mu \mathrm{m}$.

\section{Introduction}

Colloidal semiconductors and metals have been synthesized using coordinating nonaqueous media by manipulating capping ligands, ligand-solvent pairs, reactant concentration, or synthesis temperature [1-5]. $\mathrm{CuInS}_{2}$ (termed as CIS hereafter), which is a ternary chalcopyrite compound, has demonstrated more optically and electronically tunable properties than the binary II-VI analogues with the cubic zinc blend structure. CIS has recently been considered to be a promising candidate for photovoltaic applications, owing to its relatively high absorption coefficient and excellent energy matching between its band gap $(1.5 \mathrm{eV})$ and the solar spectrum. CIS nanocrystals have been synthesized by processes such as the elemental solvothermal technique $[6,7]$, thermolysis $[8,9]$, the hot injection technique $[10,11]$, and the single-source precursor route $[12,13]$. All of those techniques require high temperature and/or high pressure environments in order to bring the various kinds of species into the desirable crystal form. However, compared with the significant progress in monodisperse binary chalcopyrite colloids, investigation of ternary chalcopyrite colloids has been limited, owing to the lack of suitable synthesis methods. Therefore, the challenge remains in the preparation of monodisperse ternary chalcopyrite colloids with manageable size and shape.

The ternary chalcopyrite semiconductors expressed as IIII-VI $I_{2}$ are considered to be a superstructure of the zincblende type. In particular, $\mathrm{CuAB}_{2}(\mathrm{~A}=\mathrm{Al}, \mathrm{Ga}, \mathrm{In} ; \mathrm{B}=\mathrm{S}, \mathrm{Se})$ has shown intrinsic p-type conductivity, which suggests that chalcopyrite compounds might be interesting host materials for magnetic doping. CIS nanocrystals have been found to exhibit magnetic properties in a limited number of reports $[14,15]$. Taking these findings into consideration, we hypothesized that a new synthesis technology could be advanced by magnetic doping, in which CIS nanocrystal nucleation and growth could be self-manipulated via magnetically induced heating of the developing CIS nanocrystals upon synthesis. This is in contrast to the currently existing time-consuming, cost-ineffective, and eco-unfriendly autoclaving synthesis.

Here, we report a novel methodology for the synthesis of CIS nanocrystals in coordinating solvents without sequential chalcogenide precursor injection, which is achieved by incorporation of magnetic $\mathrm{Zn}$ species, that is, diethyldithiocarbamic zinc (DECZn), following a high frequency magnetic 
field induction (HFMF) at ambient conditions. A series of Zn-doped CIS (termed as Zn-CIS) nanocrystal-based solar cell devices using the nanocrystals with various shapes were also prepared to evaluate their performances.

\section{Experimental Procedure}

2.1. Chemicals. Copper (I) chloride $(\mathrm{CuCl}, 95 \%$, analytical reagent), indium (III) iodide ( $\left.\mathrm{InCl}_{3}, 98 \%, \mathrm{AR}\right)$, and trioctylphosphine (TOP, 90\%, technical grade) were purchased from Sigma-Aldrich Corp.; octadecene (ODE, 90\%, technical grade), oleylamine (70\%, technical grade), and diethyldithiocarbamic acid zinc salt $\left(\left[\left(\mathrm{C}_{2} \mathrm{H}_{5}\right)_{2} \mathrm{NCSS}\right]_{2} \mathrm{Zn}\right.$, technical grade $)$ were purchased from Tokyo Chemical Industry Co., Ltd.

2.2. Synthesis of $\mathrm{Zn}$-CIS Nanocrystals. Following our previous report [16], $0.5 \mathrm{mmol}$ diethyldithiocarbamic acid zinc salt was dissolved in $6 \mathrm{~mL}$ TOP. The solution was diluted with $24 \mathrm{~mL}$ ODE to form a clear solution (solution 1). Then, $0.2 \mathrm{mmol}$ $\mathrm{CuCl}$ and $\mathrm{InCl}_{3}$ wAS dissolved in $6 \mathrm{~mL}$ oleylamine at $50^{\circ} \mathrm{C}$ to form another solution (solution 2). Here, amine coordinates the $\mathrm{Cu}$ and $\mathrm{In}$ ions to produce amine complexes. These two material solutions were mixed to produce a raw material solution. A small aliquot of raw material solution was put into a test tube and exposed to HFMF. Zn-CIS nanocrystals were formed once the color turns to yellow from turbid; the input power was $90 \mathrm{~W}$.

2.3. Characterization. HFMF was set up from power supply, functional generator, amplifier, and cooling water. Similar equipment was also reported in PNAS, vol. 103, 3540-3545 (2006). The strength of the magnetic field depended on the coils. In this study, the coil is 8 loops, frequency is $50 \mathrm{kHz}$, and the strength of magnetic field $(H)$ is $2.5 \mathrm{kA} / \mathrm{m}$. The temperature of HFMF generator was controlled by cycling cooling water at $25^{\circ} \mathrm{C}$. X-ray diffraction (XRD) was carried out on a M18XHF diffractometer (MAC Science, Tokyo, Japan) with $\mathrm{Cu} \mathrm{K} \alpha_{1}$ radiation $(\lambda=0.15405 \mathrm{~nm})$ at the operation conditions of $40 \mathrm{kV}, 200 \mathrm{~mA}, 2 \theta: 10^{\circ}-70^{\circ}$, and scanning rate: $10^{\circ} / \mathrm{min}$. Transmission electron microscope (TEM) images were obtained using a JEOL 2100 transmission electron microscope operating at $200 \mathrm{kV}$. X-ray photoelectron spectroscopy (XPS) measurements were carried out using a Field Emission-Auger Electron Microprobe (Thermo VG Microlab 350) X-ray photoelectron spectrometer using an $\mathrm{Mg} \mathrm{K} \alpha \mathrm{X}$-ray as the excitation source. Then UV-vis, PL emission and excitation (PLE) spectroscopy was applied using a UV-vis spectrophotometer (UV-1600; Agelent 8453) and a spectrofluorometer (FP-6600; Jasco, Inc., Japan).

2.4. Fabrication of Zn-CIS Thin Film Solar Cells. The Mo coated soda lime glass substrates used here were fabricated by dc magnetron sputtering at Ar pressures 1.5 mTorr resulting in a $200 \mathrm{~nm}$ layer. Deposition of the CIS absorber layer on top of the Mo substrates is used drop casting by the nanoink solution and subsequent thermal treatments to remove the organics and sinter the films under Ar and Se atmospheres at $500^{\circ} \mathrm{C}$, respectively. A $\sim 50 \mathrm{~nm}$ CdS layer is then deposited by

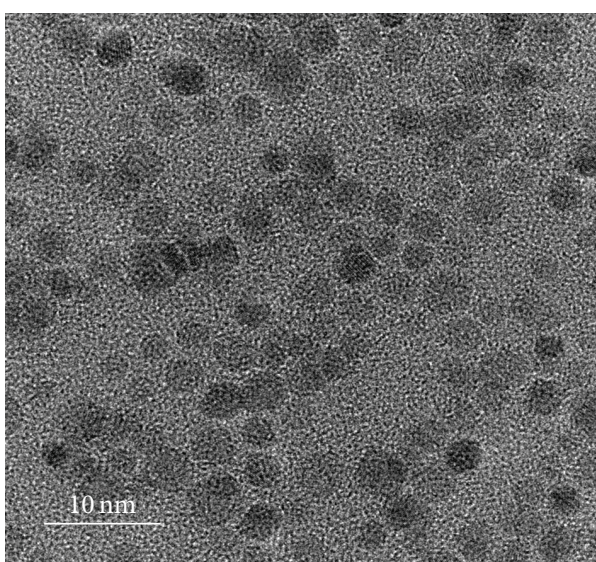

FIGURE 1: TEM image of Zn-CIS nanoparticles.

a chemical bath deposition (CBD) technique. The CBD bath contains $183 \mathrm{~mL}$ of deionized $\mathrm{H}_{2} \mathrm{O}, 25 \mathrm{~mL}$ of $0.015 \mathrm{M} \mathrm{CdSO}_{4}$ solution, $12.5 \mathrm{~mL}$ of $1.5 \mathrm{M}$ thiourea solution, and $31.25 \mathrm{~mL}$ of stock $\mathrm{NH}_{4} \mathrm{OH}$ (Aldrich). Next, A $\sim 50 \mathrm{~nm}$ high resistance intrinsic zinc oxide ( $\mathrm{i}-\mathrm{ZnO}$ ) film capped with a $\sim 300 \mathrm{~nm}$ high conductivity indium tin oxide (ITO) layer is deposited by RF magnetron sputtering. The $\mathrm{ZnO}$ film is sputtered in a mixture of $10 \% \mathrm{O}_{2}$ in Ar at sputtering pressure of $10 \mathrm{mTorr}$ without intentional heating. The ITO layer is sputtered with neither $\mathrm{O}_{2}$ nor intentional heating at sputtering pressure of $1 \mathrm{mTorr}$. After sputtering of the oxide layers, the final device is baked in air at $200^{\circ} \mathrm{C}$ over night.

\section{Results and Discussion}

Lower-magnification TEM observation was performed to monitor the formation of $\mathrm{Zn}$-CIS nanocrystals. As shown in Figure 1, spherical particles dominantly appeared in the solution in the $60 \mathrm{~s}$ sample. The TEM image of these $\mathrm{Zn}$-doped nanocrystals showed narrowly size-distributed nanoparticles, with an average diameter of $3.5 \mathrm{~nm}$. TEM image (Figure 2(a)) further indicated that these nanoparticles were single crystalline and spherical in shape. The size-specific quantum confinement of this size of the Zn-CIS nanocrystals gave a red-color solution. However, after only two minutes of magnetic field exposure, these nanocrystals grew to a size larger than the Wannier-Mott bulk exciton radius (i.e., $4.1 \mathrm{~nm}$ for CIS). To identify the crystal growth of the Zn-doped chalcopyrite semiconductor under magnetic exposure, solution samples were taken after magnetic induction for durations of 180,300 , and $420 \mathrm{~s}$, where a steady-state development of the resulting nanocrystals is assumed, albeit unintentionally selected. For the 180-second duration, the colloidal Zn-CIS nanocrystals displayed a rectangular geometry, with a size of $12-15 \mathrm{~nm}$ in length (Figure 2(b)). In the TEM images, lattice fringes corresponding to $\left\{\begin{array}{lll}0 & -1 & 1\end{array}\right\}$ and $\left\{\begin{array}{lll}2 & 2 & 0\end{array}\right\}$ CIS planes were predominantly visible. Based on these observations, the structure of the $\mathrm{Zn}$-CIS nanocrystals after $180 \mathrm{~s}$ of magnetic induction was confirmed to be trigonal-pyramidal, rather than trigonal-plate, and has been formulated for $\mathrm{CuInSe}_{2}$ and CdS. After $300 \mathrm{~s}$ of induction, a nanocubic geometry 


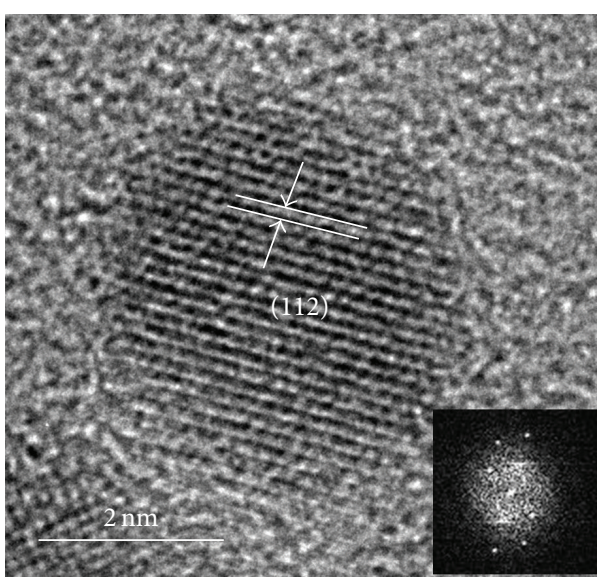

(a)

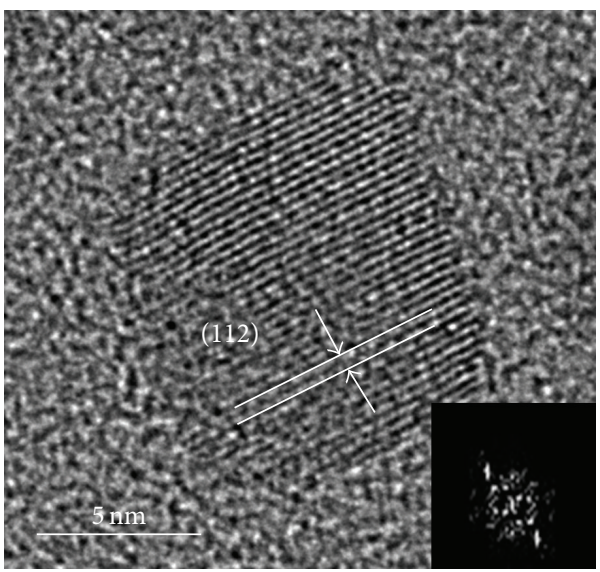

(c)

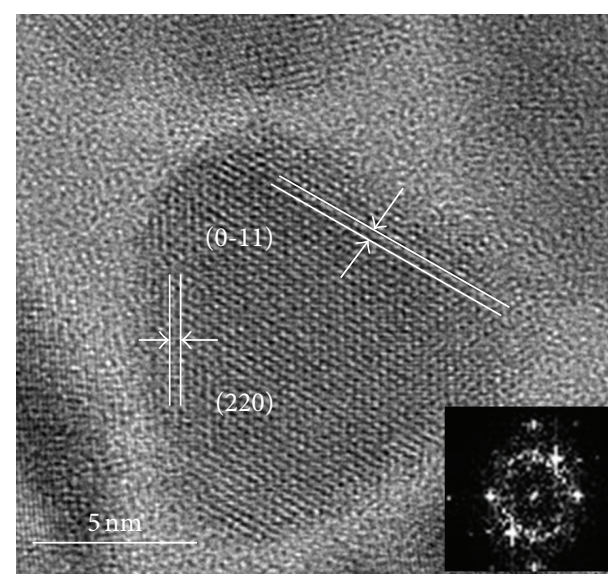

(b)

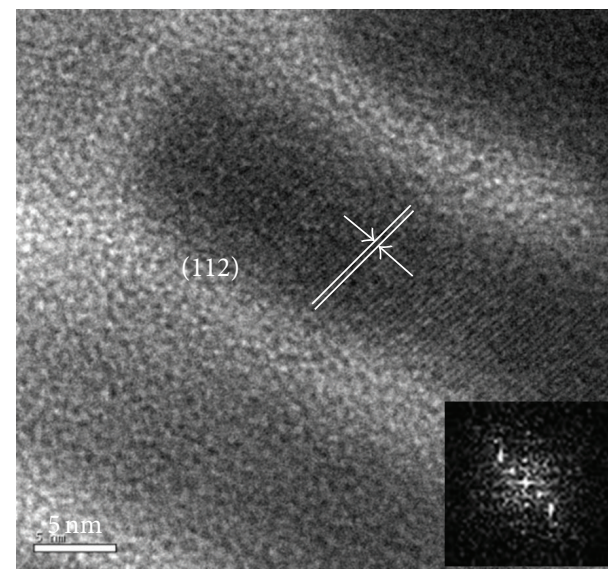

(d)

FIGURE 2: HRTEM images of the Zn-CIS nanocrystals with various geometries; (a) nanoparticle, (b) nanopyramid, (c) nanocube, and (d) nanobar synthesized under magnetic exposure.

was obtained (from the same batch of solution), as shown in Figure 2(c). The lattice fringes are separated by a distance of $3.1 \AA$, which corresponds to the $\left\{\begin{array}{lll}1 & 1 & 2\end{array}\right\}$ planes of CIS. The surface tension values of the $\left\{\begin{array}{lll}1 & 0 & 0\end{array}\right\},\left\{\begin{array}{lll}0 & 1 & 0\end{array}\right\}$, and $\left\{\begin{array}{lll}0 & 0 & 1\end{array}\right\}$ planes of nanocubes are very similar, resulting in a similar distance between these three crystallographic faces and Wulff's point. On this basis, a higher average growth rate along those crystallographic directions is expected, resulting in Zn-CIS nanocrystals evolved into rectangular or quasicubic geometry. Nevertheless, after $420 \mathrm{~s}$ of magnetic induction, the $\left\{\begin{array}{lll}1 & 1 & 2\end{array}\right\}$ plane of the nanocube showed the fastest growth rate to form a bar-like geometry with an average length of $75.4 \mathrm{~nm}$ and width of $18.3 \mathrm{~nm}$ (Figure 2(d)).

The molar ratio of $\mathrm{Zn}: \mathrm{Cu}: \mathrm{In}: \mathrm{S}$ in the nanocrystals of varying stages of magnetic induction was determined by inductively coupled plasma (ICP) spectroscopy and TEMEDX analysis. Both data indicated that a relatively uniform compositional evolution of these $\mathrm{Zn}$-CIS nanocrystals can be achieved.

To employ the (Zn-CIS) nanocrystals in solar-cell practical application, we prepared a series of Zn-CIS nanocrystalbased solar cell devices using the nanocrystals of various

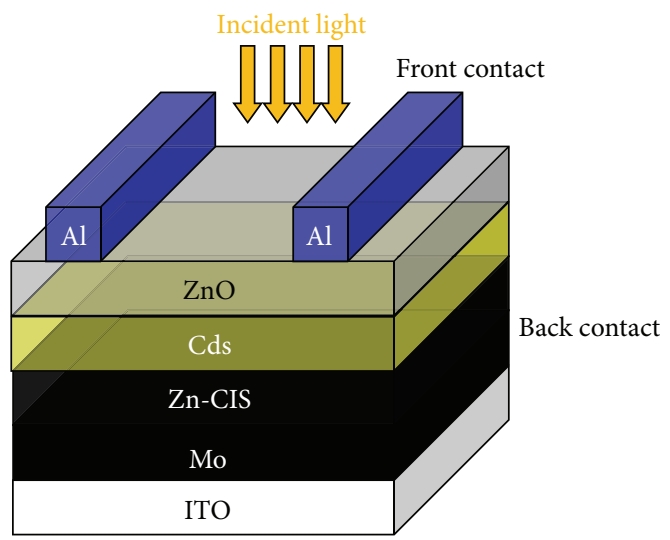

FIGURE 3: Schematic diagram of the structure of Zn-CIS device.

shapes, to measure the solar-cell parameters. The prototype device is schematically represented in Figure 3, and the current-voltage characteristics of the nanocrystal-based devices are shown in Figure 4. The relevant solar-cell parameters for those three samples are given in Table 1 , which include 


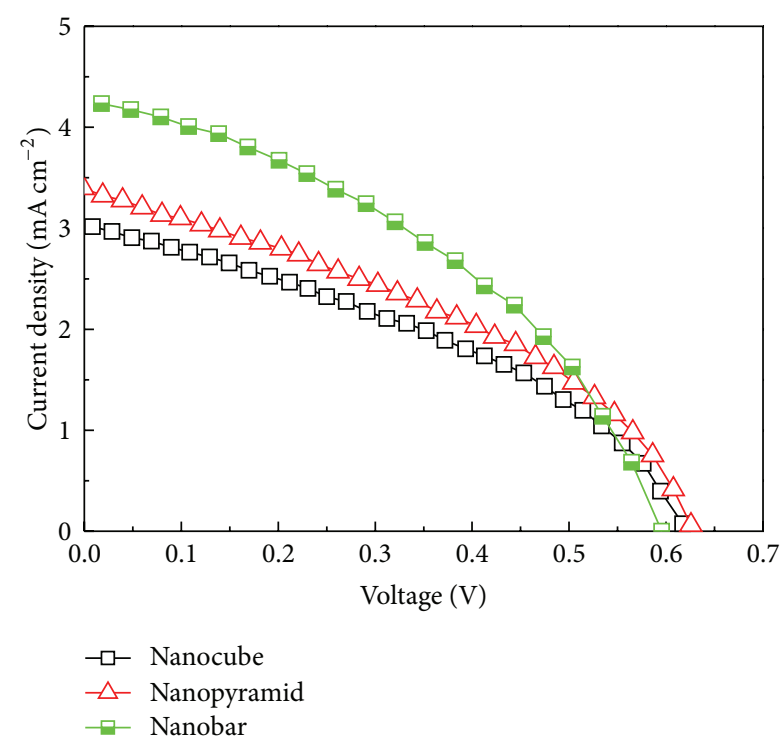

FIGURE 4: Current-voltage characteristics of Zn-CIS devices with different shape nanocrystal under stimulated AM 1.5 solar illumination $\left(0.1 \mathrm{~W} / \mathrm{cm}^{2}\right)$ and active area $0.28 \mathrm{~cm}^{2}$.

TABLE 1: Photovoltaic performance of the different shape $\mathrm{Zn}$ CIS nanocrystal-based devices under AM 1.5 solar illumination $\left(100 \mathrm{~mW} / \mathrm{cm}^{2}\right)$ and active area $0.28 \mathrm{~cm}^{2}$.

\begin{tabular}{lccc}
\hline & Nanocube & Nanopyramid & Nanobar \\
\hline$J_{\mathrm{sc}} / \mathrm{mA} \mathrm{cm}^{-2}$ & 3.012 & 3.317 & 4.21 \\
$V_{\mathrm{oc}} / \mathrm{V}$ & 0.61 & 0.62 & 0.59 \\
$\mathrm{FF}$ & 0.38 & 0.39 & 0.41 \\
$\eta(\%)$ & 0.7 & 0.80 & 1.01 \\
\hline
\end{tabular}

the current density at short circuit $\left(J_{\mathrm{sc}}\right.$ in $\left.\mathrm{mA} \mathrm{cm}^{-2}\right)$, the voltage at open circuit $\left(V_{\mathrm{oc}}\right.$ in $\left.\mathrm{V}\right)$, the fill factor $(\mathrm{FF})$, and the efficiency of power conversion $\left(\eta=J_{\text {sc }} \cdot V_{\text {oc }} \cdot \mathrm{FF} / P_{\text {in }}\right.$ with $P_{\text {in }}$ $=100 \mathrm{~mW} \mathrm{~cm}^{-2}$ ).

Nanocube-based device displayed the following characteristics: $J_{\mathrm{sc}}=3.012 \mathrm{~mA} / \mathrm{cm}^{2}, V_{\mathrm{oc}}=0.61 \mathrm{~V}, \mathrm{FF}=0.38$, and an efficiency of $0.70 \%$ on average. The $J_{\text {sc }}$ values increased from 3.012 to $3.317 \mathrm{~mA} / \mathrm{cm}^{2}$ at nanopyramid-based device accompanied with increasing efficiency from 0.7 to $0.80 \%$. The maximum value of $J_{\mathrm{sc}}$ reached $4.21 \mathrm{~mA} / \mathrm{cm}^{2}$ at nanobar-based device with a power conversion efficiency of $1.01 \%$ in this study. This result indicated that the ability of light-absorption of Zn-CIS nanocrystal was enhanced once the crystal size was increased. It is obviously suggested that the increasing efficiency of these devices is related to the broadened absorption wavelength of larger $\mathrm{Zn}$-CIS nanocrystals. The absorption band of Zn-CIS nanocrystals shows a red shift with increasing size, as shown in Figure 5. As can be seen in Table 1, compared to other parameters such as $V_{\mathrm{oc}}$ and $\mathrm{FF}$, the $J_{\mathrm{sc}}$ is more determinative for this trend, corresponded to the absorbance spectrum. However, the highest value $\left(4.21 \mathrm{~mA} / \mathrm{cm}^{2}\right)$ of $J_{\mathrm{sc}}$ here is still lower than reported $\left(17 \mathrm{~mA} \mathrm{~cm}^{-2}\right)$, which might result from thinner absorber layer. Therefore, we deposited the $\mathrm{Zn}$-CIS layers of different thickness for prototype devices,

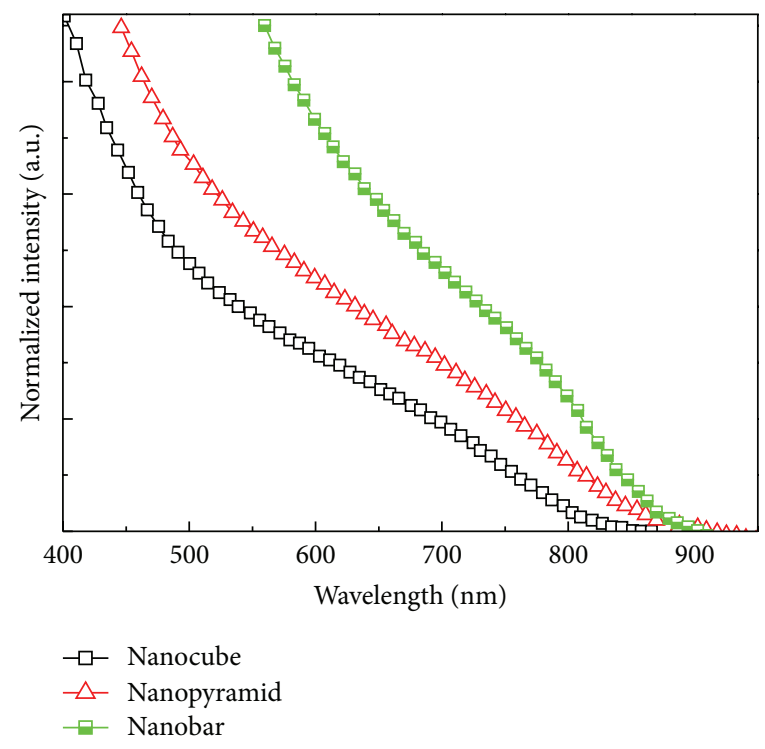

FIGURE 5: UV-vis absorption spectra of different shape Zn-CIS nanocrystals.

TABLE 2: Photovoltaic performance of the different thickness of Zn-CIS nanocrystal film devices under AM 1.5 solar illumination $\left(100 \mathrm{~mW} / \mathrm{cm}^{2}\right)$ and active area $0.28 \mathrm{~cm}^{2}$.

\begin{tabular}{lccc}
\hline Thickness $(\mu \mathrm{m})$ & 1.012 & 1.594 & 2.132 \\
$J_{\text {sc }} / \mathrm{mA} \mathrm{cm}^{-2}$ & 4.21 & 4.959 & 5.871 \\
$V_{\mathrm{oc}} / \mathrm{V}$ & 0.59 & 0.61 & 0.62 \\
$\mathrm{FF}$ & 0.41 & 0.41 & 0.39 \\
$\eta(\%)$ & 1.01 & 1.25 & 1.44 \\
\hline
\end{tabular}

in order to enhance efficiency of the device by increasing $J_{\text {sc }}$. (The $\mathrm{Zn}$-CIS nanocrystals used here are a nanobar-like sample.)

The thickness of $\mathrm{Zn}$-CIS layer can be controlled by varying the nanocrystal concentration in the suspensions. Figure 6 shows the relationship between absorber layer thickness and the efficiency (the thickness here measured by $\alpha$ step analysis). As summarized in Table 2, after increasing the thickness of Zn-CIS film, the $V_{\text {oc }}$ value shows a slight increase from 0.59 to $0.62 \mathrm{~V}$ which is related to the amount of $\mathrm{Zn}$ in the $\mathrm{Zn}$-CIS layer. The amount of $\mathrm{Zn}$ in the nanobarlike nanocrystal is lightly more than that in other two kinds of nanocrystals, which might provide explanation to the variation of the $V_{\mathrm{oc}}$. The $\mathrm{FF}$ value shows a decline from 0.41 to 0.39 at $2.132 \mu \mathrm{m}$, indicating the increasing charge recombination with thicker Zn-CIS absorber film. The source of charge recombination might have been a result of film cracking, which became more significant for thicker films than for those two thinner ones. The results display a notably systematic trend for $J_{\mathrm{sc}}$, such that the current density increases significantly from $J_{\mathrm{sc}}=4.21 \mathrm{~mA} / \mathrm{cm}^{2}$ at $1.012 \mu \mathrm{m}$ to $J_{\mathrm{sc}}=5.871 \mathrm{~mA} / \mathrm{cm}^{2}$ at $2.132 \mu \mathrm{m}$ because thicker absorber offers a promotion of light absorption and photocarrier collection. Because the extent of the increase in $J_{\mathrm{sc}}$ was much greater than the extent of the decrease in FF, the overall 


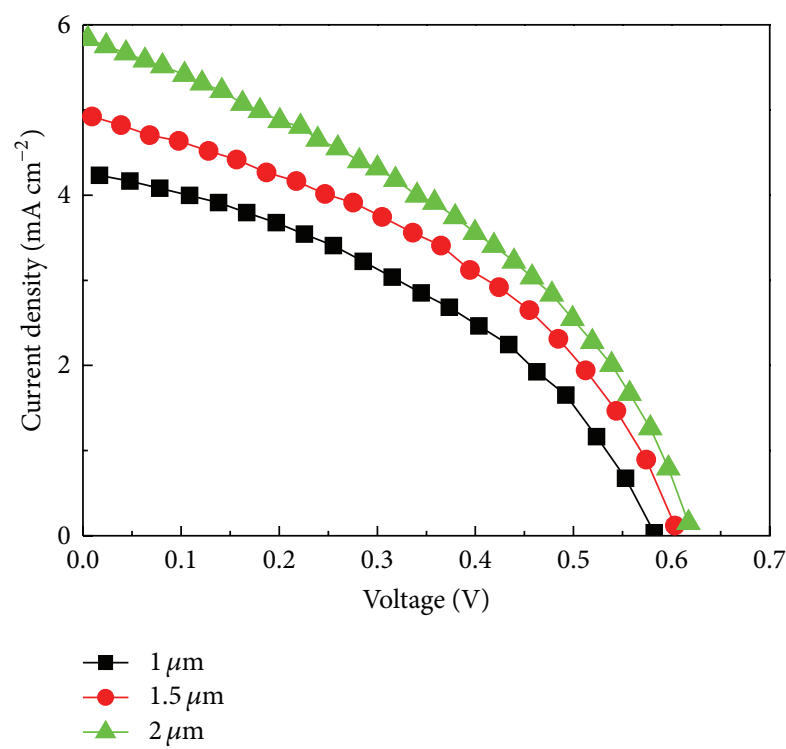

FIgURE 6: Current-voltage characteristics of Zn-CIS devices with different Zn-CIS film thickness under stimulated AM 1.5 solar illumination $\left(0.1 \mathrm{~W} / \mathrm{cm}^{2}\right)$ and active area $0.28 \mathrm{~cm}^{2}$.

efficiency of conversion of photons to current exhibits a systematic increase from $\eta=1.01 \%$ at $1.012 \mu \mathrm{m}$ to $\eta=1.44 \%$ at $2.132 \mu \mathrm{m}$.

These devices provide a baseline performance and demonstrate as a proof-of-concept that these nanocrystals can be used in PVs. Practical devices, however, require higher efficiencies. There are many ways to promote PV efficiency, including using nanocrystals with shorter chain capping ligands, incorporation of Ga into the films, and using various chemical or thermal treatment of nanocrystal layers to increase their conductivity. New device architectures that are more favorable to using nanocrystal absorber layers and low-temperature manufacturing steps may also provide ways to increase device efficiency and eliminate the need for high temperature processing. These are all important topics for further study.

\section{Conclusion}

In this thesis, we study the synthesis of magnetic-induced synthesized Zn-CIS nanocrystals. All these nanocrystals with variety in shapes are monodispersed and highly crystalline, indicating convenience, rapidity, and novelty of our method. This result demonstrates the fast crystal growth under magnetic-induced heating of such magnetic dopant chalcopyrite semiconductors has potential for energy application. A series of solar cell devices using Zn-CIS nanocrystals of various shapes were fabricated by nanoink process. The best efficiency we obtained is $1.01 \%$ for the nanobar-based device, with $J_{\mathrm{sc}}=4.12 \mathrm{~mA} / \mathrm{cm}^{2}, V_{\mathrm{oc}}=0.59 \mathrm{~V}$, and $\mathrm{FF}=0.41$. The thickness of absorber layer in these devices is around 0.983$1.012 \mu \mathrm{m}$. The efficiency of performance is promoted to $1.41 \%$ after deposition of thicker absorber layer $(2.132 \mu \mathrm{m})$.

\section{References}

[1] X. Peng, L. Manna, W. Yang et al., "Shape control of CdSe nanocrystals," Nature, vol. 404, no. 6773, pp. 59-61, 2000.

[2] V. F. Puntes, D. Zanchet, C. K. Erdonmez, and A. P. Alivisatos, "Synthesis of hcp-Co nanodisks," Journal of the American Chemical Society, vol. 124, no. 43, pp. 12874-12880, 2002.

[3] N. Cordente, C. Amiens, B. Chaudret, M. Respaud, F. Senocq, and M.-J. Casanove, "Chemisorption on nickel nanoparticles of various shapes: influence on magnetism," Journal of Applied Physics, vol. 94, no. 10, pp. 6358-6365, 2003.

[4] C. Qian, F. Kim, L. Ma, F. Tsui, P. Yang, and J. Liu, "Solution-phase synthesis of single-crystalline iron phosphide nanorods/nanowires," Journal of the American Chemical Society, vol. 126, no. 4, pp. 1195-1198, 2004.

[5] D. V. Talapin, R. Koeppe, S. Götzinger et al., "Highly emissive colloidal CdSe/CdS heterostructures of mixed dimensionality," Nano Letters, vol. 3, no. 12, pp. 1677-1681, 2003.

[6] Y. Jiang, Y. Wu, X. Mo, W. Yu, Y. Xie, and Y. T. Qian, "Elemental solvothermal reaction to produce ternary semiconductor $\mathrm{CuInE}_{2}(\mathrm{E}=\mathrm{S}, \mathrm{Se})$ Nanorods," Inorganic Chemistry, vol. 39, pp. 2964-2965, 2000.

[7] J. Xiao, Y. Xie, Y. Xiong, R. Tang, and Y. Qian, "A mild solvothermal route to chalcopyrite quaternary semiconductor $\mathrm{CuIn}\left(\mathrm{Se}_{x} \mathrm{~S}_{1-x}\right)_{2}$ nanocrystallites," Journal of Materials Chemistry, vol. 11, no. 5, pp. 1417-1420, 2001.

[8] S. L. Castro, S. G. Bailey, R. P. Raffaelle, K. K. Banger, and A. F. Hepp, "Nanocrystalline chalcopyrite materials $\left(\mathrm{CuInS}_{2}\right.$ and $\mathrm{CuInSe}_{2}$ ) via low-temperature pyrolysis of molecular singlesource precursors," Chemistry of Materials, vol. 15, no. 16, pp. 3142-3147, 2003.

[9] S. L. Castro, S. G. Bailey, R. P. Raffaelle, K. K. Banger, and A. F. Hepp, "Synthesis and characterization of colloidal $\mathrm{CuInS}_{2}$ nanoparticles from a molecular single-source precursor," Journal of Physical Chemistry B, vol. 108, no. 33, pp. 12429-12435, 2004.

[10] L. Tian, H. I. Elim, W. Ji, and J. J. Vittal, "One-pot synthesis and third-order nonlinear optical properties of $\mathrm{AgInS}_{2}$ nanocrystals," Chemical Communications, no. 41, pp. 4276-4278, 2006.

[11] K. K. Banger, M. H.-C. Jin, J. D. Harris, P. E. Fanwick, and A. F. Hepp, "A new facile route for the preparation of singlesource precursors for bulk, thin-film, and nanocrystallite I-IIIVI semiconductors," Inorganic Chemistry, vol. 42, no. 24, pp. 7713-7715, 2003.

[12] T. Kamatani and H. Akai, "The magnetic properties in transition metal-doped chalcopyrite semiconductors," Materials Science in Semiconductor Processing, vol. 6, pp. 389-391, 2003.

[13] L. H. Ye, A. J. Freeman, and B. Delley, "Half-metallic ferromagnetism in $\mathrm{Cu}$-doped $\mathrm{ZnO}$ : density functional calculations," Physical Review B, vol. 73, no. 3, Article ID 033203, 4 pages, 2006.

[14] B. Koo, R. N. Patel, and B. A. Korgel, "Synthesis of CuInSe nanocrystals with trigonal pyramidal shape," Journal of the American Chemical Society, vol. 131, no. 9, pp. 3134-3135, 2009.

[15] N. Pinna, K. Weiss, J. Urban, and M. P. Pileni, "Triangular CdS nanocrystals: structural and optical studies," Advanced Materials, vol. 13, no. 4, pp. 261-264, 2001.

[16] K. T. Kuo, S. H. Hu, D. M. Liu, and S. Y. Chen, "Magneticallyinduced synthesis of highly-crystalline ternary chalcopyrite nanocrystals under ambient conditions," Journal of Materials Chemistry, vol. 20, pp. 1744-1750, 2010. 

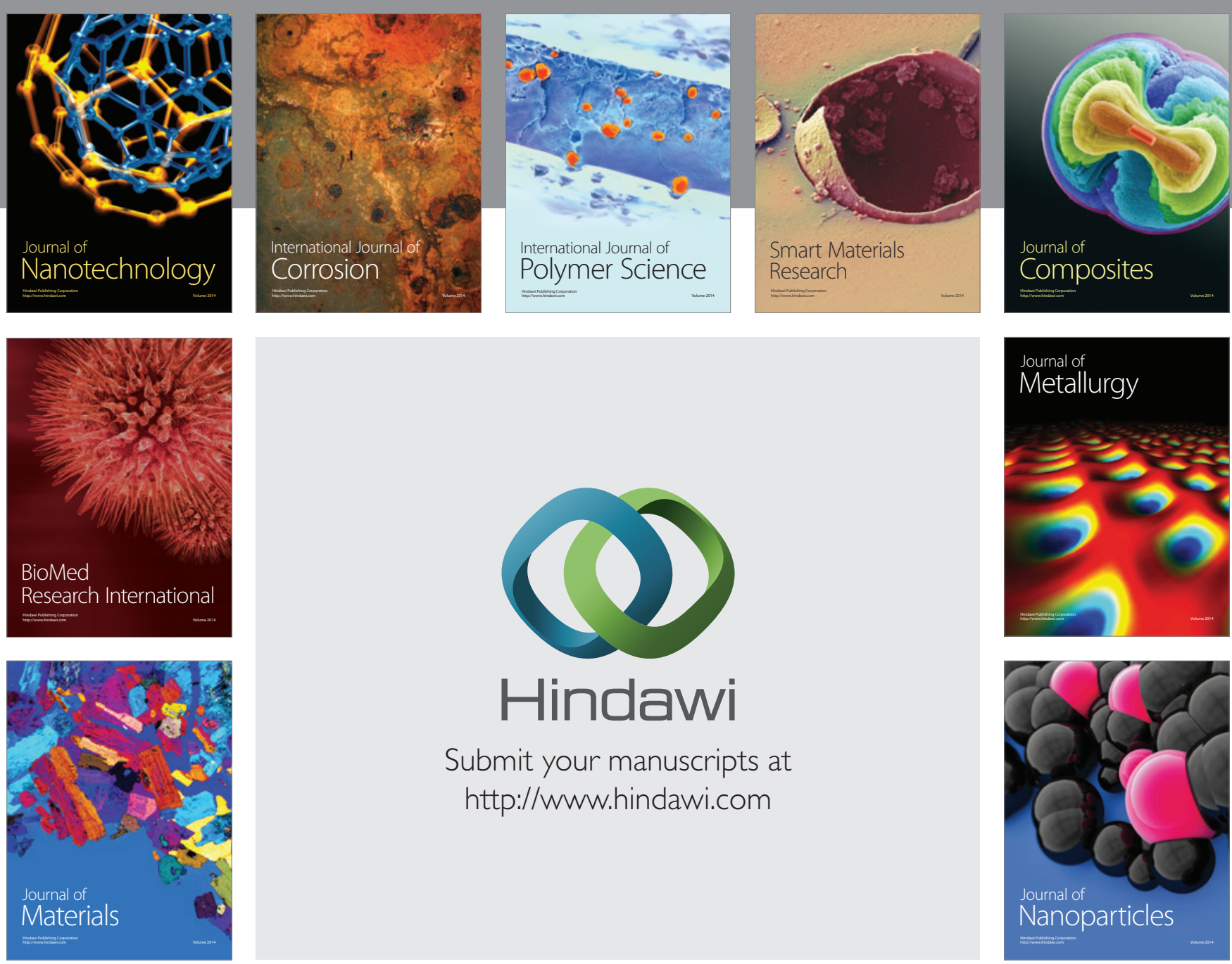

Submit your manuscripts at http://www.hindawi.com
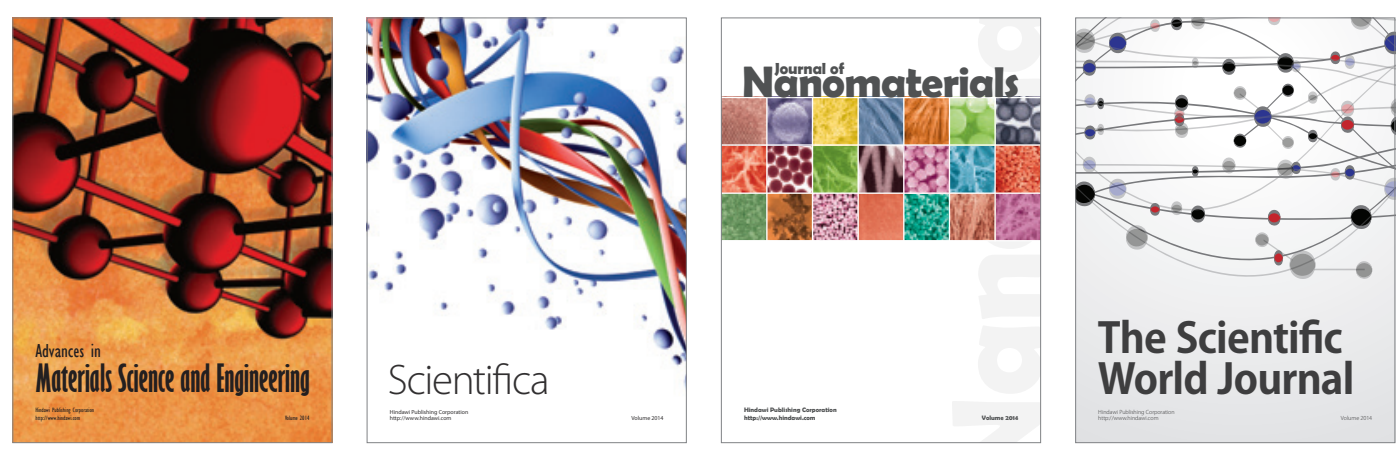

\section{The Scientific World Journal}
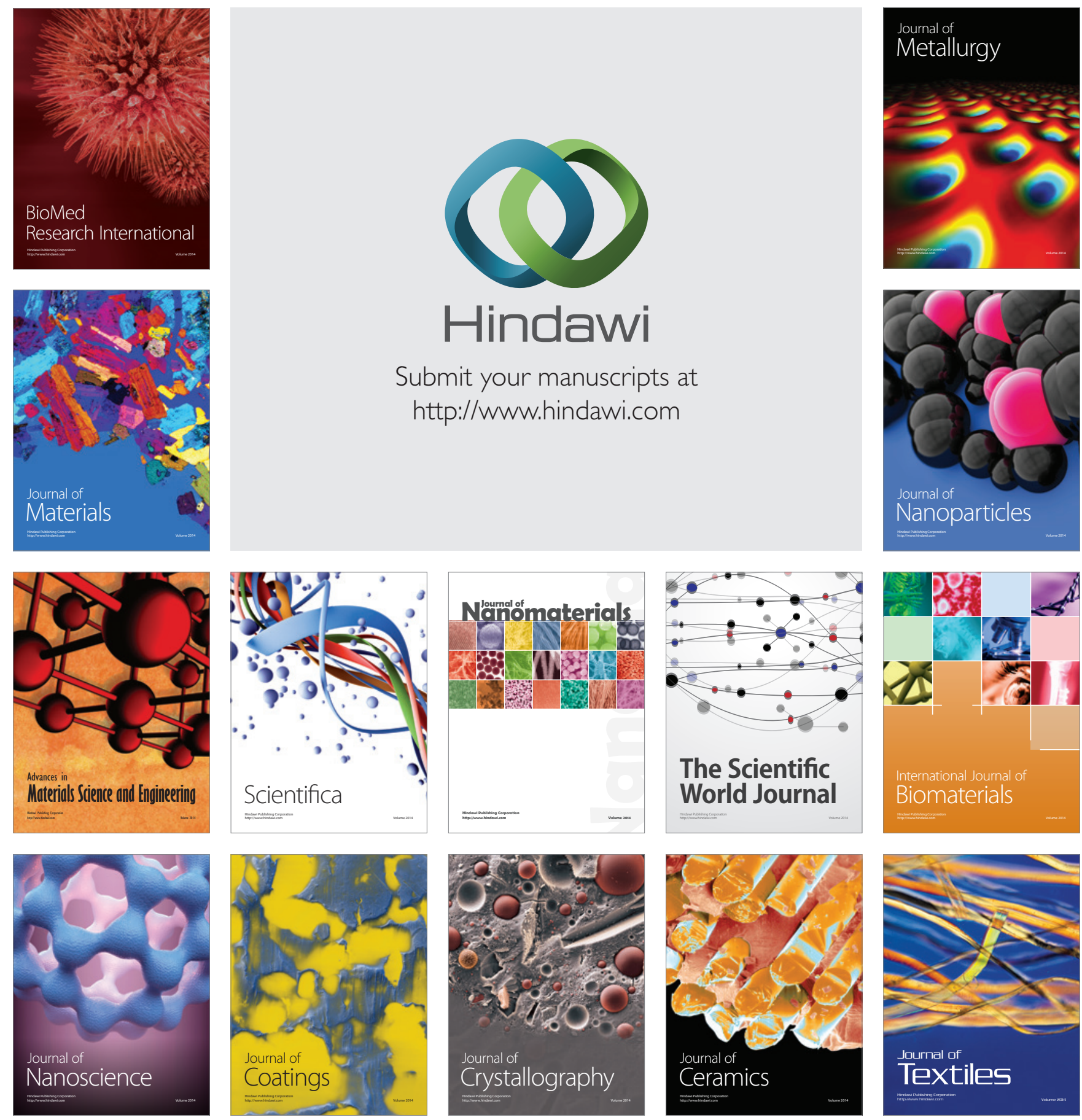